\title{
From zero resistance states to absolute negative conductivity in microwave irradiated two-dimensional electron systems
}

\author{
J. Iñarrea ${ }^{a)}$ \\ Escuela Politécnica Superior, Universidad Carlos III, Leganes, Madrid 28911, Spain \\ and Instituto de Ciencia de Materiales, CSIC, Cantoblanco, Madrid 28049, Spain \\ G. Platero \\ Instituto de Ciencia de Materiales, CSIC, Cantoblanco, Madrid 28049, Spain
}

(Received 15 May 2005; accepted 19 June 2006; published online 2 August 2006)

\begin{abstract}
Recent experimental results regarding a two-dimensional electron gas subjected to microwave radiation reveal that magnetoresistivity, apart from presenting oscillations and zero resistance states, can evolve to negative values at minima. Here the authors present a theoretical model which explains the transition from zero resistance states to absolute negative conductivity in terms of multiphoton assisted electron scattering due to charged impurities and shows how this transition can be driven by tuning microwave frequency and intensity. This opens the possibility of controlling the magnetoconductivity in microwave driven nanodevices and understanding the novel optical and transport properties of such devices. (C) 2006 American Institute of Physics.
\end{abstract}

[DOI: $10.1063 / 1.2335408]$

The effect of an ac field on the electronic transport properties of nanostructures has been an active research topic in the last years. Ten years ago, transport experiments on acdriven weakly coupled semiconductor superlattices reveled a fascinating, nonintuitive, behavior: for certain parameters of the ac potential and stationary electric field, the electronic current flowed uphill presenting absolute negative conductivity (ANC). ${ }^{1}$ Recently two experimental groups ${ }^{2,3}$ have announced the existence of oscillations and zero resistance states (ZRSs) in the longitudinal magnetoresistivity $\left(\rho_{x x}\right)$ of two-dimensional electron systems (2DESs) subjected to microwave (MW) radiation and a perpendicular magnetic field $(B)$. One of the most controversial topics in this field has been the existence of ANC and even the very presence of ZRS has been also questioned. ${ }^{4,5}$ Most experimental works, ${ }^{2,3,6-10}$ report clearly such vanishing dissipation states but not ANC. Only Willett et al., ${ }^{11}$ and very recently Zudov et al., ${ }^{12}$ have reported minima in which $\rho_{x x}$ is distinctly negative. On the other hand many theoretical contributions have been presented to explain $\rho_{x x_{5}}$ oscillations with $B$ and the possibility of ZRS and ANC.,5,13-21

Here we present a microscopical theory which explains how the system evolves from ZRS to ANC. The proposed theory is based on a recently presented model ${ }^{19}$ where we explain the $\rho_{x x}$ behavior of a 2DES at low $B$ and under MW radiation. We obtained the exact solution of the corresponding electronic wave function, which is expressed is terms of photosatellites and energy sidebands,

$$
\begin{aligned}
\Psi(x, t)= & \phi_{n}\left[x-X-x_{\mathrm{cl}}(t), t\right] \exp \left[i \frac{m^{*}}{\hbar} \frac{d x_{\mathrm{cl}}(t)}{d t}\left[x-x_{\mathrm{cl}}(t)\right]\right. \\
& \left.+\frac{i}{\hbar} \int_{0}^{t} L d t^{\prime}\right] \times \sum_{m=-\infty}^{\infty} J_{m}\left[\frac { e E _ { 0 } } { \hbar } X \left(\frac{1}{w}\right.\right. \\
& \left.\left.+\frac{w}{\sqrt{\left(w_{c}^{2}-w^{2}\right)^{2}+\gamma^{4}}}\right)\right] e^{i m w t},
\end{aligned}
$$

where $e$ is the electron charge, $\phi_{n}$ is the solution for the Schrödinger equation of the unforced quantum harmonic oscillator, $w$ the MW frequency, $w_{c}$ the cyclotron frequency, $E_{0}$ the intensity for the MW field, $X$ is the center of the orbit for the electron motion, $x_{\mathrm{cl}}(t)$ is the classical solution of a forced harmonic oscillator, $x_{\mathrm{cl}}=e E_{o} / m^{*} \sqrt{\left(w_{c}^{2}-w^{2}\right)^{2}+\gamma^{4}} \cos w t, L$ is the lagrangian, and $J_{m}$ are Bessel functions. According to that model, due to the MW radiation, center positions of electronic orbits are not fixed, but they oscillate back and forth harmonically with $w$. The amplitude $A$ for these harmonic oscillations is given by $A=e E_{o} / m^{*} \sqrt{\left(w_{c}^{2}-w^{2}\right)^{2}+\gamma^{4}}$.

Electrons suffer scattering due to charged impurities that are randomly distributed in the sample. To proceed we calculate the electron-charged impurity multiphoton assisted transition rate $W_{n, m}$ from an initial state $\Psi_{n}(x, t)$ to a final state $\Psi_{m}(x, t)$ :

$W_{n, m}=W_{n, m}(0) \times\left[B_{0}+B_{1}+B_{2}\right]$, where $W_{n, m}(0)$ is the transition rate when $J_{0} \simeq 1$ (see Ref. 19), and

$$
\begin{aligned}
B_{0}= & {\left[J_{0}^{2}\left(A_{m}\right) J_{0}^{2}\left(A_{n}\right)+\sum_{i=1}^{s} 2 J_{i}^{2}\left(A_{m}\right) J_{i}^{2}\left(A_{n}\right)\right] } \\
& \times\left[\frac{\Gamma}{\left[\hbar w_{c}(n-m)\right]^{2}+\Gamma^{2}}\right], \\
B_{1}= & {\left[\sum_{i=0}^{s} J_{i}^{2}\left(A_{m}\right) J_{i+1}^{2}\left(A_{n}\right)+J_{i+1}^{2}\left(A_{m}\right) J_{i}^{2}\left(A_{n}\right)\right] } \\
& \times\left[\frac{\Gamma}{\left[\hbar w_{c}(n-m)+\hbar w\right]^{2}+\Gamma^{2}}\right], \\
B_{2}= & {\left[J_{1}^{2}\left(A_{m}\right) J_{1}^{2}\left(A_{n}\right)+\sum_{i=0}^{s} J_{i}^{2}\left(A_{m}\right) J_{i+2}^{2}\left(A_{n}\right)\right.} \\
& \left.+J_{i+2}^{2}\left(A_{m}\right) J_{i}^{2}\left(A_{n}\right)\right]\left[\frac{\Gamma}{\left[\hbar w_{c}(n-m)+2 \hbar w\right]^{2}+\Gamma^{2}}\right] .
\end{aligned}
$$

${ }^{a)}$ Electronic mail: jesusinarrea@icmm.csic.es 


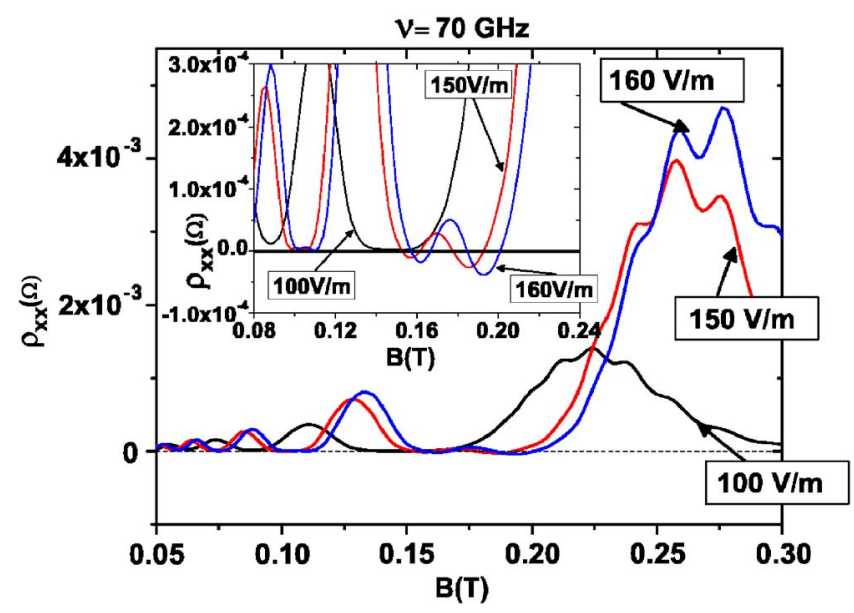

FIG. 1. (Color online) Calculated magnetoresistivity $\rho_{x x}$ as a function of $B$, for different MW intensities at $\nu=70 \mathrm{GHz}$. In the inset we show amplifications of principal minima. Zero resistance states, absolute negative conductivity, and the corresponding evolution for increasing MW power can be observed. Temperature: $500 \mathrm{mK}$.

$$
\text { Here } \quad A_{m,(n)}=\left[\left(e E_{0} / \hbar\right) X_{m,(n)}(1 / w\right.
$$
$\left.+w / \sqrt{\left.\left(w_{c}^{2}-w^{2}\right)^{2}+\gamma^{4}\right)}\right] e^{i m w t}$. The summation index $i$ is the number of sidebands considered, which fixes the highest order of multiphoton processes taken into account. In our calculation we have considered up to two photons. $\Gamma$ is the state (Landau level) broadening.

The next step is to find out the average effective distance advanced by the electron in every scattering jump which is given by (see Ref. 19 for a detailed explanation): $\Delta X^{\mathrm{MW}}$ $=\Delta X^{0}+A \cos w \tau$, where $\Delta X^{0}$ is the effective distance advanced when there is no MW field present and $1 / \tau=W_{n, m}(\tau$ being the impurity scattering time). If the average value $\Delta X^{\mathrm{MW}}$ is different from zero over all the scattering processes, the electron possesses an average drift velocity $v_{n, m}$ in the $x$ direction. This drift velocity can be calculated readily by introducing the term $\Delta X^{\mathrm{MW}}$ into the transition rate, and finally the longitudinal conductivity $\sigma_{x x}$ can be written as $\sigma_{x x}=\left(2 e / E_{\mathrm{dc}}\right) \int \rho\left(E_{n}\right) v_{n, m}\left[f\left(E_{n}\right)-f\left(E_{m}\right)\right] d E_{n}$, where $f_{i}$ and $f_{f}$ are the distribution functions for the initial and final Landau states, respectively. Gathering all the terms, we finally obtain the expression

$$
\begin{aligned}
\sigma_{x x}= & \sigma_{x x}(0)\left[B_{0}\left[f\left(E_{n}\right)-f\left(E_{m}\right)\right]+B_{1}\left[f\left(E_{n}\right)-f\left(E_{m}+\hbar w\right)\right]\right. \\
& \left.+B_{2}\left[f\left(E_{n}\right)-f\left(E_{m}+2 \hbar w\right)\right]\right],
\end{aligned}
$$

and $\sigma_{x x}(0)$ is the conductivity when $J_{0} \simeq 1$ (see Ref. 19). To obtain $\rho_{x x}$ we use the relation $\rho_{x x}=\sigma_{x x} /\left(\sigma_{x x}^{2}+\sigma_{x y}^{2}\right) \simeq \sigma_{x x} / \sigma_{x y}^{2}$, where $\sigma_{x y} \simeq n_{i} e / B$ and $\sigma_{x x} \ll \sigma_{x y}$.

In Fig. 1 the calculated $\rho_{x x}$ as a function of $B$ for different MW powers at fixed frequency $\nu=w / 2 \pi=70 \mathrm{GHz}$ is shown. The inset shows the evolution from ZRS to negative conductivity. Our results follow the qualitative experimental behavior $^{11}$ and show that ANC occurs at the principal minimum, as the incident radiation power is increased. The physical explanation is as follows. In Fig. 2 we represent schematic diagrams to describe $\rho_{x x}$ evolution at minima. In Fig. 2(a) orbits are moving forward and on average the electron advances a shorter distance than in the no MW case, $\Delta X^{\mathrm{MW}}<\Delta X^{0}$. This corresponds to a decrease in the conductivity but $\rho_{x x}>0$ still. If we raise the MW power we will eventually reach the situation depicted in Fig. 2(b), where orbits are moving forwards but their amplitude $A$ is larger Downloaded 12 Mar 2010 to 161.111 .180 .191 . Redistribution subje

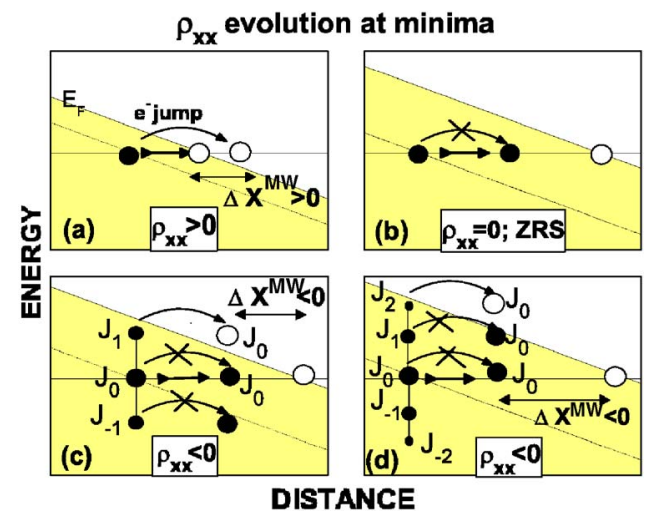

FIG. 2. (Color online) Schematic diagrams of electronic transport describing $\rho_{x x}$ evolution at minima, for fixed MW frequency and different MW intensities.

than the electronic jump. In this case the jump is blocked because the final state is occupied. This is the physical origin of the ZRS. At fixed photon frequency, small values for MW power correspond to transitions where no photon absorption or emission is involved, i.e., the arguments of Bessel functions are so small that only $J_{0}$ terms need to be taken into account. This implies that only direct, $J_{0} \rightarrow J_{0}$, transitions are relevant. If we further increase the MW power, while keeping the frequency constant, the argument of the Bessel functions will become larger, and higher order sidebands must be considered: multiphoton transitions become relevant. Transitions such as $J_{1} \rightarrow J_{0}$ and $J_{2} \rightarrow J_{0}$, which correspond to one photon and two photon processes, respectively, can then play an effective role in the current. At minima and for $A$ larger than the electron scattering jump (large MW power), we find a situation where $\Delta X^{\mathrm{MW}}<0$. However, for multiphoton transitions $J_{1} \rightarrow J_{0}$ or $J_{2} \rightarrow J_{0}$, etc., the difference of distribution functions $\left(f_{i}-f_{f}\right)>0$. These processes $\left(\Delta X^{\mathrm{MW}}<0\right.$ and $\left(f_{i}\right.$ $\left.\left.-f_{f}\right)>0 \Rightarrow \rho_{x x}<0\right)$ produce negative contributions to the current and are the physical origin of ANC. One surprising effect in the experimental results is the positive peak in the middle of $\rho_{x x}$ negative minimum. This is also observed in calculated results (see inset of Fig. 1). The explanation for this can be readily obtained. In a regime with $\Delta X^{\mathrm{MW}}<0$ and finite temperature, direct $\left(J_{0} \rightarrow J_{0}\right)$ transitions can correspond to negative values for the difference of electronic distribution functions $\left(f_{i}-f_{f}\right)$. This is due to the fact that in such a regime, the final state is always deeper in energy than the initial state, with respect to the Fermi energy. Considering the smearing of the distribution function at finite temperature, the final result is that $f_{i}<f_{f} \Rightarrow\left(f_{i}-f_{f}\right)<0$. When $\Delta X^{\mathrm{MW}}<0$ and $\left(f_{i}-f_{f}\right)<0$, an effective positive net current will be produced giving rise to a positive $\rho_{x x}$. Positive net values for $\rho_{x x}$ in the middle of the main minima have been experimentally obtained by other groups, ${ }^{7,11}$ notably in Ref. 7, where this effect was termed "breakdown of ZRS."

Figure 3 shows $\rho_{x x}$ vs $B$ for different MW frequencies at fixed MW power. ANC is achieved for all the cases presented and the growth of the central peak as frequency is increased is clearly visible (see inset). The explanation is as follows. At constant $E_{0}$, lower $w$ corresponds to high values for the Bessel function arguments, i.e., $J_{0}$ 's are decreasing and $J_{n \neq 0}$ 's are increasing. In this regime, direct transitions (positive contributions) become less important than multiphoton, $J_{n} \rightarrow J_{0}$ transitions (negative contributions). Eventuto AIP license or copyright; see http://apl.aip.org/apl/copyright.jsp 


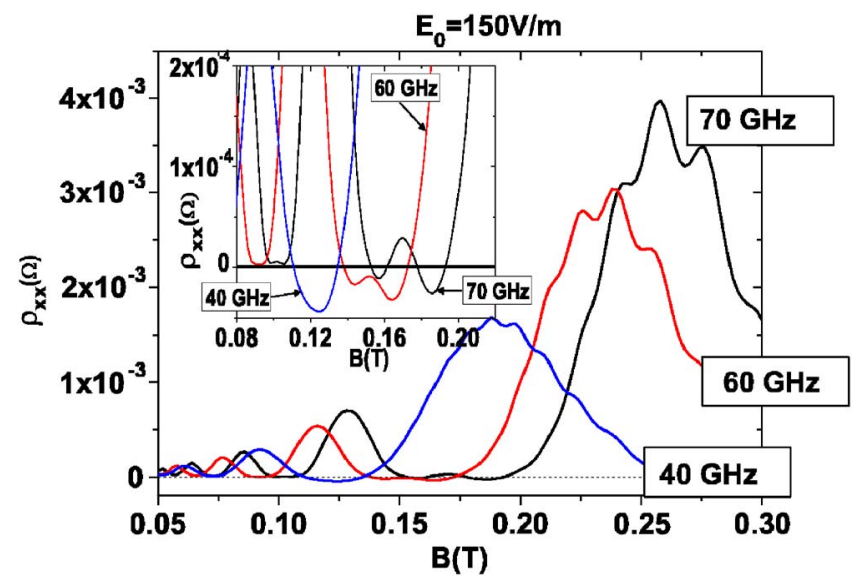

FIG. 3. (Color online) Calculated magnetoresistivity $\rho_{x x}$ as a function of $B$, for different frequencies at MW intensity $E_{0}=150 \mathrm{~V} / \mathrm{m}$. In the inset we display a rising central peak in the middle of negative minimum that becomes positive as the MW frequency is increased. Temperature: $500 \mathrm{mK}$.

ally the positive contributions are totally compensated by the negative ones and the central peak at minimum vanishes.

In Fig. 4(a) we represent calculated $\rho_{x x}$ vs $B$ at fixed $w$ and $E_{0}$ and at increasing external dc bias $E_{\mathrm{dc}}$. We can observe at the two main minima how the $\rho_{x x}$ negative values are shifted to positive as $E_{\mathrm{dc}}$ is raised. The explanation can be seen from the schematic diagrams in Figs. 4(b) and 4(c). In both cases we are in a regime where $\Delta X^{\mathrm{MW}}<0$. At lower bias $\left[E_{\mathrm{dc}}\right.$, Fig. $\left.4(\mathrm{~b})\right]$, one-photon transitions giving negative current $\left(J_{1} \rightarrow J_{0}\right)$ dominate and, except in the central peak, they can compensate the positive direct $\left(J_{0} \rightarrow J_{0}\right)$ contributions. We observe that the final state for $J_{1} \rightarrow J_{0}$ is well above the Fermi energy and the corresponding difference $\left(f_{i}-f_{f}\right)$ $>0$ is important making the negative contributions relevant.
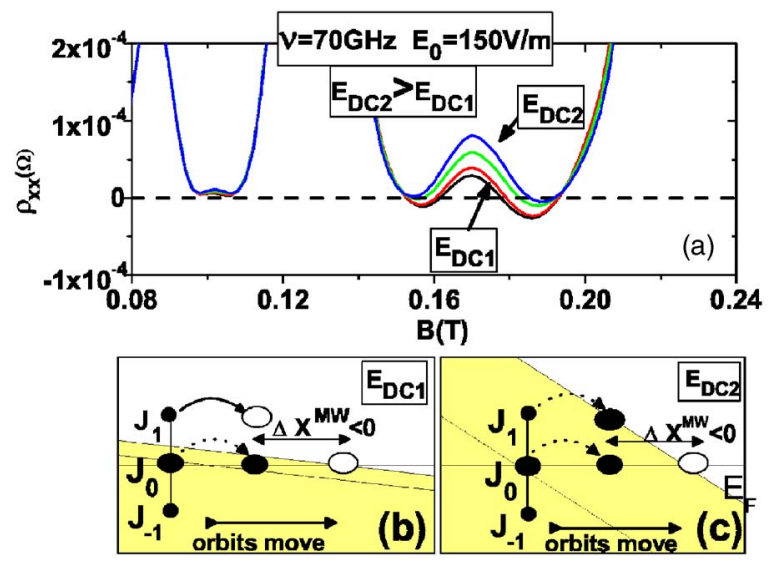

FIG. 4. (Color online) (a) Calculated $\rho_{x x}$ vs $B$ at two minima for fixed MW frequency and intensity and different external dc biases $\left(E_{\mathrm{dc}}\right)$. Temperature $500 \mathrm{mK}$.
At higher bias $E_{\mathrm{dc} 2}$, we reach the situation depicted in Fig. 4(c). The corresponding slope for the Fermi energy is larger than in the $E_{\mathrm{dcl}}$ case. Now the final state is below $E_{F}$, i.e., it is not empty making the corresponding difference $\left(f_{i}-f_{f}\right)$ $<0$ and the contribution to the current evolves from negative to positive. The final result is that the $\rho_{x x}$ negative values get smaller for increasing $E_{\mathrm{dc}}$ becoming eventually zero or positive.

Therefore we can state that high values for $E_{0} / w$ and a regime of lower values for $E_{\mathrm{dc}}$, constitute the two main conditions which have to be fulfilled in order to obtain ANC. Thus according to our theoretical model and taking into account of the experimental difficulties to measure negative resistance, we believe that by tuning the appropriate MW parameters and external dc bias, it would be possible to experimentally observe the evolution from ZRS to ANC.

The authors acknowledge Charles Creffield for the critical reading of this letter. This work was supported by the MCYT (Spain) Grant No. MAT2005-06444, the "Ramon y Cajal" program to one of the authors (J.I.), and the EU Human Potential Programme HPRN-CT-2000-00144.

${ }^{1}$ B. J. Keay, S. J. Allen, J. Galan, J. P. Kaminski, K. L. Chapman, A. C. Gossard, U. Battacharya, and M. J. W. Rodwell, Phys. Rev. Lett. 75, 4098 (1995).

${ }^{2}$ R. G. Mani, J. H. Smet, K. von Klitzing, V. Narayanamurti, W. B. Johnson, and V. Umansky, Nature (London) 420, 646 (2002).

${ }^{3}$ M. A. Zudov, R. R. Du, N. Pfeiffer, and K. W. West, Phys. Rev. Lett. 90, 046807 (2003)

${ }^{4}$ A. C. Durst, S. Sachdev, N. Read, and S. M. Girvin, Phys. Rev. Lett. 91, 086803 (2003)

${ }^{5}$ A. V. Andreev, I. L. Aleiner, and A. J. Millis, Phys. Rev. Lett. 91, 056803 (2003).

${ }^{6}$ R. G. Mani, V. Narayanamurti, K. von Klitzing, J. H. Smet, W. B. Johnsonand, and V. Umansky, Phys. Rev. B 69, 161306(R) (2004).

${ }^{7}$ R. G. Mani, Appl. Phys. Lett. 85, 4962 (2004).

${ }^{8}$ S. A. Studenikin, M. Potemski, A. Sachrajda, M. Hilke, L. N. Pfeiffer, and K. W. West, Phys. Rev. B 71, 245313 (2005).

${ }^{9}$ C. L. Yang, M. A. Zudov, T. A. Knuuttila, R. R. Du, L. N. Pfeiffer, and K. W. West, Phys. Rev. Lett. 91, 096803 (2003).

${ }^{10}$ J. H. Smet, B. Gorshunov, C. Jiang, L. Pfeiffer, K. West, V. Umansky, M. Dressel, R. Dressel, R. Meisels, F. Kuchar, and K. von Klitzing, Phys. Rev. Lett. 95, 116804 (2005).

${ }^{11}$ R. L. Willett, L. N. Pfeiffer, and K. W. West, Phys. Rev. Lett. 93, 026804 (2004).

${ }^{12}$ M. A. Zudov, R. R. Du, L. N. Pfeiffer, and K. W. West, Phys. Rev. B 73, 041303 (2006).

${ }^{13}$ C. Joas, J. Dietel, and F. von Oppen, Phys. Rev. B 72, 165323 (2005).

${ }^{14}$ X. L. Lei and S. Y. Liu, Phys. Rev. B 72, 075345 (2005).

${ }^{15}$ V. Ryzhii and V. Vyurkov, Phys. Rev. B 68, 165406 (2003).

${ }^{16}$ Junren Shi and X. C. Xie, Phys. Rev. Lett. 91, 086801 (2003).

${ }^{17}$ Kang-Hun Ahn, J. Korean Phys. Soc. 47, 666 (2005).

${ }^{18}$ A. V. Andreev, I. L. Aleiner, and A. J. Millis, Phys. Rev. Lett. 91, 056803 (2003).

${ }^{19}$ J. Iñarrea and G. Platero, Phys. Rev. Lett. 94, 016806 (2005).

${ }^{20}$ T.-K. Ng and Lixin Dai, Phys. Rev. B 72, 235333 (2005).

${ }^{21}$ J. Iñarrea and G. Platero, Phys. Rev. B 72, 193414 (2005). 